\title{
Essai comparatif de l'utilisation des extraits du Neem et du virus entomopathogène MaviNPV dans la gestion des insectes ravageurs du niébé en milieu paysan au Niger
}

\author{
Maimouna ABDOURAHAMANE HAROUNA ${ }^{1,2}$, Ibrahim BAOUA $^{2 *}$, Sitou LAWALI $^{2}$, \\ Manuele TAMÒ ${ }^{3}$, Laouali AMADOU ${ }^{1}$, Saadou MAHAMANE ${ }^{4}$ et Barry PITTENDRIGH ${ }^{5}$
}

\author{
${ }^{I}$ Institut National de la Recherche Agronomique du Niger (INRAN) BP 240 Maradi Niger. \\ ${ }^{2}$ Université Dan Dicko Dankoulodo de Maradi, BP 465 Maradi, Niger. \\ ${ }^{3}$ Institut International d'Agriculture Tropicale (IITA), BP 0932 Cotonou, Bénin. \\ ${ }^{4}$ Université Abdou Moumouni de Niamey BP 10662, Niamey, Niger. \\ ${ }^{5}$ Department of Entomology, Michigan State University, East Lansin MI 44824 USA. \\ *Auteur correspondant; E-mail: baoua.ibrahim@gmail.com
}

\section{RESUME}

Le niébé (Vigna unguiculata (L.) Walp (Fabaceae) est une culture importante dont le rendement est faible en raison de fortes attaques des insectes ravageurs. Cette étude a été entreprise pour comparer l'efficacité de cinq biocides sur la gestion des principaux insectes ravageurs dans la région de Zinder au niveau de quatre villages en 2014 et vingt-cinq villages en 2015. Un dispositif en blocs de Fischer à deux répétitions et six traitements a été mis en place avec trois producteurs par village. Pour les deux années, le pesticide chimique de synthèse et les extraits aqueux de Neem ont été les plus performants avec 6,1 à 17,1\% des gousses présentant des dégâts de Clavigralla tomentosicollis Stål, 3,3 à 7,0\% affectées par Maruca vitrata Fabricius et des rendements en graines variant de 811 à $1233 \mathrm{~kg} / \mathrm{h}$. Les traitements à l'huile de Neem, au mélange TopBio + Virus et au Virus ont connu plus d'infestation que les traitements précédents avec 23,8 à 65,9\% des gousses attaquées par $C$. tomentosicollis, 17,6 à 25,6\% affectées par $M$. vitrata et un rendement de 389 à $616 \mathrm{~kg} / \mathrm{ha}$. Les résultats obtenus encouragent l'utilisation des extraits aqueux de graines de Neem en milieu paysan comme solution de lutte alternative contre les principaux insectes ravageurs du niébé.

(C) 2019 International Formulae Group. All rights reserved

Mots clés: Biopesticide, Clavigralla tomentosicollis, Maruca vitrata, perte, rendement.

\section{Comparative test for the use of Neem extract and entomopathogenic virus MaviNPV for the management of cowpea insect pests in Niger rural area}

\begin{abstract}
Cowpea (Vigna unguiculata (L.) Walp (Fabaceae) is an important crop with low yield due to serious damage by insect pests. This study was conducted to compare the efficacy of five biocides to control the major insect pests in four villages during 2014 and in twenty-five villages during 2015 in Zinder region. A Fischer block design with two repetitions and six treatments was established with three farmers per village. The synthetic chemical pesticide and Neem aqueous extracts were the most effective for both years with 6.1 to $17.1 \%$ pods
\end{abstract}


damaged by Clavigralla tomentosicollis Stål ; 3.3 to $7.0 \%$ by Maruca vitrata Fabricius and the seed yields ranged from 811 to $1233 \mathrm{~kg} / \mathrm{ha}$. The Neem oil, TopBio + Virus and Virus treatments recorded more infestation than the previous treatment with 23.8 to $65.9 \%$ of the pods infested by Clavigralla tomentosicollis Stål , 17.6 to 25.6 infested by Maruca vitrata Fabricius and yield of 389 to $616 \mathrm{~kg} / \mathrm{ha}$. The results obtained encourage the use of Neem seed aqueous extracts in rural area as an alternative control method against the cowpea main insect pests. (C) 2019 International Formulae Group. All rights reserved

Keywords: Biopesticide, Clavigralla tomentosicollis, Maruca vitrata, losses, yield.

\section{INTRODUCTION}

Le niébé (Vigna unguiculata (L.) Walp (Fabaceae) est une légumineuse à grains importante dans les régions tropicales et subtropicales (Oyewale et Bamaiyi, 2013). En effet, le niébé est un aliment de base apprécié en Afrique car ses feuilles, ses gousses vertes et ses graines sèches peuvent être consommées et commercialisées. La graine mûre contient 23-25\% de protéine, 50-67\% d'amidon, des vitamines B tel que l'acide folique qui est important dans la prévention de malformation chez le nouveau-né. La graine est également riche en micro-éléments essentiels, tels que le fer, le calcium et le zinc (Cisse et Hall, 2002). Le niébé est généralement préféré par les agriculteurs en raison de son rôle dans l'augmentation de la fertilité du sol par la fixation de l'azote (Asiwe, 2006). Le Niger second pays producteur mondial après le Nigeria, occupe une superficie en niébé estimée à 5858085 ha en 2017 avec une production d'environ 1953707 tonnes en 2017 (MA, 2018). Cependant, sa production est confrontée à des contraintes qui limitent son rendement dont entre autres les précipitations irrégulières, les températures du sol élevées, la faible fertilité du sol, les mauvaises herbes parasites, les maladies et les insectes nuisibles (FAO, 2004). Les principaux insectes ravageurs du niébé répertoriés dans le monde et occasionnant des dégâts économiques considérables sur la plante sont: Maruca vitrata Fabricius (Lepidoptera : Crambidae), Megalurothrips sjostedti Trybom (Thysanoptera : Thripidae), Clavigralla tomentosicollis Stål (Heteroptera : Coreidae) et Aphis craccivora Koch (Homoptera : Aphididae) (Liao et Lin, 2000 ; Egho, 2011). La pression des ravageurs est l'une des causes expliquant la faible productivité de la culture avec un rendement de $368 \mathrm{~kg} / \mathrm{ha}$ en moyenne dans la région de Zinder (MA, 2018), rendement insignifiant par rapport au potentiel de la plante, estimé à trois tonnes/ha (Asiwe ,2006 ; Tanzubil et al., 2008).

La punaise brune, $C$. tomentosicollis Stål en particulier est responsable des dégâts sur le niébé causant des pertes de rendement en grains allant de 17,5 à 26,5\% en l'absence d'une mesure de contrôle (Abdourahamane et al., 2018). M. vitrata occasionne des pertes de rendement de 30 à 86\% (Tamò et al., 2003).

$\mathrm{Au}$ Niger, dans les régions de Maradi et Zinder, selon une étude effectuée par Rabé et al., (2017), 57,2\% des paysans font recours à la lutte chimique pour augmenter leur production. Cette méthode présente plusieurs autres contraintes. Les pesticides renferment des substances dangereuses et toxiques même à très faibles doses (Carlos, 2006). Selon Gomgnimbou et al., 2009), leur utilisation est source des risques d'ordre sanitaire, de pollution des eaux et des sols et de développement de résistance chez les parasites ciblés (Ouédraogo, 2004 ; Bass et al.,2015 ; Narayan et al., 2017). Les effets des pesticides sur l'environnement et la santé ne sont donc pas compatibles avec une exploitation durable des agro-écosystèmes comme mentionné par plusieurs auteurs (Deguine et Ferron, 2004 ; Narayan et al., 2017).

Les inconvénients liés à l'emploi des pesticides de synthèse ont conduit les services phytosanitaires à concevoir une stratégie reposant sur une prévention des infestations des ravageurs. On note aussi un regain d'intérêt sur des méthodes alternatives de lutte et écologiques faisant appel à l'usage des biopesticides naturels. Les Méliacées sont les 
végétaux les plus expérimentés pour leur effet biocide. Le Neem Azadirachta indica A. Juss est l'espèce la plus étudiée dans ce cadre (Facknath, 2006). Selon Mouffok et al. (2008), les extraits aqueux des graines renferment plus de 168 composés dont un groupe de 7 substances proches de l'azadirachtine. En zone sahélienne, l'efficacité des extraits de Neem a été démontrée pour la protection phytosanitaire du niébé (Bambara et Tiemtoré, 2008; Abdoulaye et al., 2018), sur le pourghère (Jatropha curcas L.) (Mominé, 2014) et la tomate (Zakari et al., 2016).

La lutte biologique par l'utilisation des entomopathogènes est une alternative très prometteuse pour la gestion intégrée des insectes ravageurs (McGurire et al., 2005). Les microorganismes utilisés sont entre autres les bactéries, les virus et les champignons ou leur sous-produits (Sabbour et Sahab, 2005 ; Reddy et al., 2008). Les virus entomopathogènes spécialement Nucleopolyhedroviruses NPV et granulovirus GV sont connus pour leur efficacité contre les insectes nuisibles d'importance agricole (Vinod et Singh, 2009). Les formulations de NPV se sont révélées efficaces contre la pyrale des légumineuses sur les légumineuses alimentaires, seules ou en association avec $B$. thuringiensis et Neem, en laboratoire et sur le terrain à Taiwan et au Bénin (Tamo et al., 2007 ; Srinivasan et al., 2008 ; Sokamé, 2012 ; Mehinto et al., 2014 ; Abdoulaye et al., 2018).

La présente étude a eu donc pour but de fournir aux paysans dans leur contexte agro écologique, des alternatives durables en termes de gestion des ravageurs par l'utilisation d'insecticides naturels à base de Neem. De façon plus spécifique, elle vise à comparer l'efficacité de quatre insecticides naturels contre les insectes nuisibles du niébé en champ en particulier la punaise suceuse des gousses, C. tomentosicollis et la foreuse de gousses, $M$. vitrata.

\section{MATERIEL ET METHODES Site de l'étude}

L'étude a été menée dans la région de Zinder (latitude : $13^{\circ} 48^{\prime} 19^{\prime}{ }^{\prime} \mathrm{N}, 08^{\circ} 59^{\prime} 18^{\prime \prime} \mathrm{E}$ ) localisée au centre-sud du Niger dans la zone sahélienne pendant les saisons hivernales 2014 et 2015. Les essais ont été mis en place sur des sols dunaires ferrugineux de couleur brun-clair, riches en sables fins dans l'horizon 0-30 cm en 2014 dans quatre villages du département de Tanout et respectivement dans cinq, onze et neufs villages des départements de Gouré, Tanout et Magaria en 2015 (Figure 1).

\section{Dispositif expérimental}

Le dispositif expérimental utilisé est un bloc de Fischer avec six traitements et deux répétitions dans trois sites installés dans chacun des villages. La parcelle élémentaire est de $6 \mathrm{~m}$ x $5 \mathrm{~m}$ espacées de $2 \mathrm{~m}$. La variété IT90K-3721-2 de l'IITA a été semée à une densité de 0,75 $\mathrm{m} \times 0,50 \mathrm{~m}$ soit 9 lignes de 11 poquets par parcelle. Les traitements suivants ont été appliqués : le jus de la graine de Neem, l'huile de la graine de Neem, la suspension du virus MaviNPV, le produit TopBio mélangé à la suspension de virus MaviNPV, le pesticide chimique de synthèse. Les parcelles témoins n'ont reçu aucune pulvérisation.

\section{Application des méthodes de traitement}

Deux applications espacées d'une semaine ont été effectuées à la formation des boutons floraux du niébé. Les substances aqueuses sont pulvérisées avec un équipement à pression entretenue d'une capacité de 20 litres et les produits huileux avec un appareil ULV à piles.

Les différentes solutions ont été préparées de la manière suivante pour chacun des villages. Les solutions obtenues ont permis le traitement des 3 sites d'un village.

- Virus MaviNPV (Virus Polyédrose Nucléaire) : il a été mélangé $115 \mathrm{ml}$ de virus à 4001 d'eau par ha (Laboratoire de la pathologie de l'IITA-Bénin)

- Jus de graines de Neem (Azadirachtine) : $12,5 \mathrm{~kg}$ de poudre d'amandes de Neem obtenus après le décorticage et le pilage ont été mélangés à 250 litres d'eau et conservé durant $24 \mathrm{~h}$. Le filtrat a été utilisé pour la pulvérisation d'un hectare (Tanzubil et al., 2008 ; Egho et Ilondu, 2012). Les applications ont été effectuées l'après-midi à partir de $16 \mathrm{~h}$; 
- Huile de Neem (Azadirachtine, la salannine, la nimbidine et le mélandriol): il a été mélangé 1,6 1 d'huile extraite des amandes de Neem à 8,71 d'huile d'arachide par hectare (BiophytoGlazoué Bénin, http://www.agrobiobase.com/fr/annuaire/b ioproduits/agriculture-sylvicultureviticulture/top-bio,)

- TopBio + virus : Le produit TopBio est une émulsion à base d'huile de Neem et d'huiles essentielles extraites de la citronnelle (Bio phyto Glazoué, Bénin, http://www.agrobiobase.com/fr/annuaire/b ioproduits/agriculture-sylviculture-

viticulture/top-bio). La solution pulvérisée a été obtenue en mélangeant 21 de TopBio à $115 \mathrm{ml}$ de virus. Le mélange a été dilué avec 401 d'eau pour la pulvérisation d'un hectare

- Insecticide chimique de synthèse Conquest C 88 EC $(16 \mathrm{~g} / 1$ de acétamipride et $72 \mathrm{~g} / 1$ de cyperméthrine): il a été mélangé $500 \mathrm{ml}$ d'insecticide de synthèse à 1001 d'eau par hectare (Saphyto S.A. BP : 1390 Bobo-Dioulasso Burkina Faso).

\section{Collecte des données}

Les données suivantes ont été collectées pour chacun des sites :

- Les infestations des ravageurs : Il a été dénombré au cours des deux années, les effectifs des insectes sur cinq poquets choisis aléatoirement et matérialisés dans chacune des parcelles élémentaires. Les observations ont eu lieu chaque semaine et ont débuté en aout 2014 et 2015. Ces observations ont été effectuées pendant six semaines avant les traitements phytosanitaires ;

- Les dégâts des ravageurs : Il a été noté la production des gousses par poquet et les gousses portant des traces des attaques de la punaise et de $M$. vitrata sur cinq poquets matérialisés dans chacune des parcelles élémentaires ;

- Le rendement par traitement: Il a été déterminé à partir des récoltes effectuées sur les deux lignes centrales. Les gousses ont été décortiquées pour la détermination du poids des graines. Le rendement a été calculé en rapportant la production obtenue sur les deux lignes à l'hectare.

\section{Analyse des données}

Le test ANOVA et SNK ont été utilisés pour comparer les infestations par ravageur, les proportions de gousses attaquées et les rendements entre les différents traitements. Les analyses ont été effectuées avec le logiciel Statistical Package for Social Science (SPSS) version 16 et le logiciel Excel.

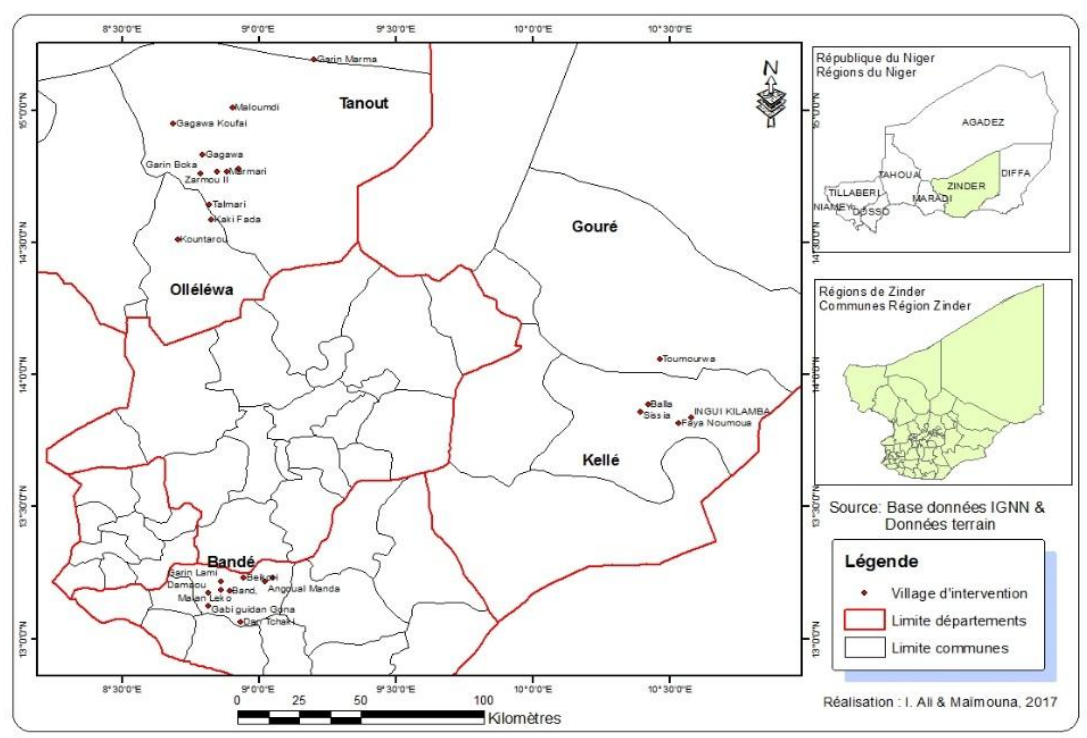

Figure 1: Carte de localisation des villages enquêtés. 


\section{RESULTATS}

La punaise brune $C$. tomentosicollis et la foreuse des gousses $M$. vitrata ont été les espèces les plus répertoriées durant la phase de fructification du niébé.

Pour les deux années, les densités de la punaise brune ont été plus élevées dans les parcelles témoins et celles traitées au virus MaviNPV comparativement aux autres traitements. Dans les parcelles traitées au pesticide et au jus de Neem, les densités du ravageur ont été 2,7 à 4,5 fois moins importantes comparativement au traitement témoin (Tableau 1). Dans les parcelles traitées à l'huile de Neem et au mélange TopBio + Virus, comparativement aux parcelles traitées au pesticide chimique, il a été noté des densités de C. tomentosicollis 2,4 fois plus élevées en 2014 et 2,9 fois plus importantes en 2015 .

En 2014 et en 2015, le nombre de larves de $M$. vitrata par cinq poquets dans les parcelles traitées au pesticide chimique et au jus de graines de Neem a été 5,0 à 7,9 fois moins important par rapport aux témoins. Les densités du ravageur ont été comparables dans les parcelles traitées à l'huile de Neem, au mélange TopBio + Virus et au Virus. Cependant, les effectifs des ravageurs dans ces deux traitements ont été 1,6 à 3,3 fois moins importants que dans les parcelles témoins et 2,0 à 5,0 fois plus élevées que dans celles traitées au jus de graines de Neem ou au pesticide.

Les proportions des gousses portant des dégâts de $C$. tomentosicollis dans les parcelles témoins ont été élevées par rapport aux autres traitements avec un ratio de $89,9 \%$ en 2014 et $74,7 \%$ en 2015. Comparativement au traitement témoin, les ratios des gousses endommagées par le ravageur sont 5,2 à 14,3 fois plus bas dans les traitements au pesticide chimique ou au jus de graines de Neem et 1,6 à 3,1 fois moins élevés dans les parcelles traitées à l'huile de Neem, au mélange TopBio + Virus et au Virus de 2014 à 2015.
Dans le traitement témoin, le ratio des gousses attaquées par M. vitrata a été de 85,0\% en 2014 et $47,3 \%$ en 2015. Les traitements au pesticide chimique ou au jus de graines de Neem ont été comparables avec des proportions des gousses portant les traces des dégâts de $M$. vitrata 11,8 à 14,2 fois moins importants par rapport au traitement témoin au cours des deux années. Les traitements à l'huile de Neem, au mélange TopBio + Virus et au Virus ont été comparables avec des proportions de gousses endommagées 2,4 à 3,7 fois moins importantes par rapport au traitement témoin.

La production des gousses a été 4,9 à 5,1 fois plus élevée en 2014 et 1,6 à 1,8 fois plus importante en 2015 dans les traitements au pesticide chimique et au jus de graines de Neem comparativement au traitement témoin (Tableau 3). En 2014, les traitements à l'huile de Neem, au mélange TopBio + Virus et au Virus MaviNPV ont donné une production des gousses 2,2 à 2,6 fois moins importante que celle du traitement au pesticide. En 2015, le traitement témoin et celui au Virus MaviNPV ont enregistré la plus faible production des gousses. Les traitements à l'huile de Neem et au mélange TopBio + Virus ont donné une production 1,3 fois plus élevée que celle du traitement témoin et 1,2 à 1,4 fois plus faible que celle des traitements au pesticide chimique et au jus de graines de Neem.

Pour la production en graine, le traitement au pesticide a été comparable à celui du jus de graines de Neem avec un rendement 2,9 à 19,9 fois plus élevé que celui des parcelles témoins pour les deux années d'expérimentation (Tableau 4). Les traitements à l'huile de Neem, au mélange TopBio + Virus et au Virus ont donné des productions en graines 1,3 à 10,3 fois plus élevées que celles des traitements témoins et 1,5 à 2,4 fois moins importantes que celles des parcelles traitées au pesticide pour les deux années. 
M. ABDOURAHAMANE HAROUNA et al. / Int. J. Biol. Chem. Sci. 13(2): 950-961, 2019

Tableau 1 : Nombre moyen des larves et adultes de C. tomentosicollis et des larves de $M$. vitrata par 5 poquets en fonction par traitement et par année.

\begin{tabular}{|c|c|c|c|c|}
\hline \multirow{2}{*}{ Traitements } & \multicolumn{2}{|c|}{ Larves et adultes de $C$. tomentosicollis } & \multicolumn{2}{|c|}{ Larves de $M$. vitrata } \\
\hline & 2014 & 2015 & 2014 & 2015 \\
\hline Insecticide chimique & $3,3 \pm 0,3 a$ & $2,7 \pm 0,8 \mathrm{a}$ & $0,8 \pm 0,1 \mathrm{a}$ & $0,3 \pm 0,0 \mathrm{a}$ \\
\hline Jus de graines de Neem & $4,1 \pm 0,4 a$ & $3,6 \pm 0,7 a$ & $0,9 \pm 0,2 \mathrm{a}$ & $0,4 \pm 0,0 \mathrm{ab}$ \\
\hline Huile de Neem & $7,6 \pm 0,7 b$ & $7,1 \pm 1,0 \mathrm{ab}$ & $2,9 \pm 0,3 \mathrm{ab}$ & $1,0 \pm 0,3 b$ \\
\hline TopBio + virus & $7,9 \pm 0,6 b$ & $8,4 \pm 2,1 \mathrm{ab}$ & $3,4 \pm 1,1 \mathrm{ab}$ & $0,6 \pm 0,1 \mathrm{ab}$ \\
\hline Virus MaviNPV & $10,0 \pm 0,5 c$ & $11,7 \pm 1,9 b$ & $4,0 \pm 1,6 b$ & $0,6 \pm 0,0 \mathrm{ab}$ \\
\hline Témoin sans pesticide & $9,9 \pm 0,7 \mathrm{c}$ & $13,7 \pm 2,8 b$ & $6,4 \pm 0,6 c$ & $2,0 \pm 0,2 \mathrm{c}$ \\
\hline ANOVA & $\mathrm{F}=24,3 ; \mathrm{df}=5 / 333 ; \mathrm{P}<0,01$ & $\mathrm{~F}=6,4 ; \mathrm{df}=5 / 617 ; \mathrm{P}=0,004$ & $\mathrm{~F}=16,29 ; \quad \mathrm{df}=5 / 333 ; \mathrm{P}<0,01$ & $\mathrm{~F}=22,0 ; \mathrm{df}=5 / 617 ; \mathrm{P}<0,01$ \\
\hline
\end{tabular}

Tableau 2 : Nombre moyen de gousses présentant des attaques de $C$. tomentosicollis et de $M$. vitrata par 5 poquets par traitement et par année.

\begin{tabular}{|c|c|c|c|c|}
\hline \multirow[t]{2}{*}{ Traitements } & \multicolumn{2}{|c|}{ Gousses présentant des attaques de $C$. tomentosicollis } & \multicolumn{2}{|c|}{ Gousses présentant des attaques de $M$. vitrata } \\
\hline & 2014 & 2015 & 2014 & 2015 \\
\hline Insecticide chimique & $1,2 \pm 0,1 \mathrm{a}$ & $1,9 \pm 0,0 \mathrm{a}$ & $1,1 \pm 0,1 \mathrm{a}$ & $0,5 \pm 0,1 \mathrm{a}$ \\
\hline Jus de graines de Neem & $1,3 \pm 0,1 \mathrm{a}$ & $2,2 \pm 0,1 \mathrm{a}$ & $1,4 \pm 0,2 \mathrm{a}$ & $0,7 \pm 0,1 \mathrm{a}$ \\
\hline Huile de Neem & $2,4 \pm 0,2 b$ & $4,1 \pm 0,3 b$ & $2,8 \pm 0,3 b$ & $2,4 \pm 0,5 b$ \\
\hline TopBio + virus & $2,4 \pm 0,3 b$ & $4,6 \pm 0,1 b$ & $2,7 \pm 0,3 b$ & $1,9 \pm 0,3 \mathrm{ab}$ \\
\hline Virus MaviNPV & $3,5 \pm 0,4 c$ & $5,8 \pm 0,4 \mathrm{c}$ & $2,6 \pm 0,2 b$ & $1,8 \pm 0,3 \mathrm{ab}$ \\
\hline Témoin sans pesticide & $3,5 \pm 0,5 \mathrm{c}$ & $6,2 \pm 0,2 c$ & $5,5 \pm 0,6 \mathrm{c}$ & $4,0 \pm 0,7 \mathrm{c}$ \\
\hline ANOVA & $F=11,2 ; \mathrm{df}=5 / 292 ; P<0,01$ & $\mathrm{~F}=67,9 ; \mathrm{df}=5 / 2630 ; \mathrm{P}<0,01$ & $\begin{array}{c}\mathrm{F}=17,5 ; \mathrm{df}=5 / 292 \\
\mathrm{P}<0,01\end{array}$ & $\mathrm{~F}=10,3 ; \mathrm{df}=5 / 2630 ; \mathrm{P}<0,01$ \\
\hline
\end{tabular}


Tableau 3 : Nombre moyen des gousses par poquet par traitement et par année.

\begin{tabular}{lcc}
\hline Traitements & $\mathbf{2 0 1 4}$ & $\mathbf{2 0 1 5}$ \\
\hline Insecticide chimique & $20,2 \pm 1,2 \mathrm{a}$ & $14,9 \pm 0,1 \mathrm{a}$ \\
Jus de graines de Neem & $19,4 \pm 1,4 \mathrm{a}$ & $13,1 \pm 0,3 \mathrm{ab}$ \\
Huile de Neem & $8,5 \pm 0,5 \mathrm{~b}$ & $11,0 \pm 0,6 \mathrm{~b}$ \\
TopBio + virus & $10,2 \pm 0,5 \mathrm{~b}$ & $11,0 \pm 1,0 \mathrm{~b}$ \\
Virus MaviNPV & $9,1 \pm 0,4 \mathrm{~b}$ & $8,7 \pm 0,7 \mathrm{c}$ \\
Témoin sans pesticide & $3,9 \pm 0,6 \mathrm{c}$ & $8,4 \pm 0,5 \mathrm{c}$ \\
ANOVA & $\mathrm{F}=45,6 ; \mathrm{df}=5 / 292 ; \mathrm{P}<0,01$ & $\mathrm{~F}=17,4 ; \mathrm{df}=5 / 2630 ; \mathrm{P}<0,01$ \\
\hline
\end{tabular}

Tableau 4 : Production moyenne des parcelles (kg/ha) par traitement et par année.

\begin{tabular}{lcc}
\hline Traitements & $\mathbf{2 0 1 4}$ & $\mathbf{2 0 1 5}$ \\
\hline Insecticide chimique & $1233,3 \pm 44,1 \mathrm{a}$ & $922,6 \pm 22,9 \mathrm{a}$ \\
Jus de graines de Neem & $1150,0 \pm 86,6 \mathrm{a}$ & $810,8 \pm 17,6 \mathrm{~b}$ \\
Huile de Neem & $616,0 \pm 34,0 \mathrm{~b}$ & $604,9 \pm 13,3 \mathrm{c}$ \\
TopBio + virus & $563,5 \pm 163,5 \mathrm{~b}$ & $562,2 \pm 9,8 \mathrm{c}$ \\
Virus MaviNPV & $518,0 \pm 82,0 \mathrm{~b}$ & $389,4 \pm 28,2 \mathrm{~d}$ \\
Témoin sans pesticide & $60,0 \pm 10,0 \mathrm{c}$ & $309,5 \pm 12,6 \mathrm{e}$ \\
ANOVA & $\mathrm{F}=26,1 ; \mathrm{df}=5 / 10 ; \mathrm{P}<0,01$ & $\mathrm{~F}=162,16 ; \mathrm{df}=5 / 345 ; \mathrm{P}<0,01$
\end{tabular}

\section{DISCUSSION}

Les données collectées dans cette étude ont permis de connaître les principaux ravageurs du niébé dans la région de Zinder. $C$. tomentosicollis semble être un ravageur important du niébé en champ. Son incidence sur la production ne semble pas négligeable. Dans les parcelles témoins non traitées, les effectifs de la punaise sont 2,7 à 4,5 fois plus importantes et plus de $2 / 3$ des gousses sont endommagées par l'insecte. Les effectifs des larves de $M$. vitrata ont été 5 à 7 fois plus importants dans les parcelles témoins par rapport à celles traitées au pesticide chimique. Pour les deux années, dans les parcelles témoins, $47 \%$ à $85 \%$ des gousses produites par la plante ont été perforées par le ravageur.

En zone sahélienne, la pression biotique est donc importante sur le niébé. Ces ravageurs utilisent les organes de fructification de la plante pour se nourrir et se reproduire affectant ainsi sa productivité (Dugjé et al., 2009 ; N'gbesso et al., 2013).

Dans la présente expérimentation, dans les parcelles témoins, il est noté un rendement de 60 à $310 \mathrm{~kg} / \mathrm{ha}$ au cours de deux années soit 
une perte de 62 à $95 \%$ par rapport aux parcelles traitées au pesticide chimique. Ce ratio de perte imputable aux insectes n'est pas loin des pertes souvent totales rapportées par certains auteurs (Habiba, 2004 ; Talekar, 2006 ; Ahmed et al., 2009 ; Tonessia et al., 2009).

Cette perte est aussi relative au poids des graines. Les graines issues des gousses attaquées présentent des altérations ce qui se traduit par la dépréciation de la qualité des récoltes (Habiba, 2004 ; Talekar, 2006 ; Tonessia et al., 2009).

Cette situation traduit ce qui se passe en milieu paysan dans cette région. Selon Rabé et al. (2017), la majorité des producteurs $(62,2 \%)$ de la région n'utilise aucune méthode de lutte et cela explique le faible rendement régional moyen du niébé qui est $368 \mathrm{Kg} / \mathrm{ha}$ (MA, 2018).

Le développement de la culture du niébé demande la diffusion et l'adoption à large échelle des méthodes de protection phytosanitaire. Dans la présente étude, il est démontré que l'utilisation de l'insecticide chimique de synthèse permet de réduire les infestations du niébé par les insectes ravageurs de $80,0 \%$ et les dégâts sur les gousses de $92,0 \%$ tout en permettant d'obtenir des rendements 2,9 à 19,9 fois supérieurs aux rendements des parcelles témoins. Les rendements de 923 à $1233 \mathrm{~kg} / \mathrm{ha}$ obtenus sur les deux années d'expérimentation avec le pesticide CONQUEST 88 EC sont proches des 859 $\mathrm{kg} / \mathrm{ha}$ obtenus avec la deltaméthrine à la dose de 11/ha dans la zone de Ziga au Burkina Faso (Bambara et Tiemtoré, 2008). D'autres études effectuées au Burkina Faso et au Bénin ont démontré que les infestations des fleurs par $M$. vitrata ont été 3,7 à 5,6 fois moins importantes dans les champs avec l'application des pesticides chimiques LAMBDACE $25 \mathrm{EC}$ et le DECIS 12,5 EC (Mehinto et al., 2014; Dieudonné et al., 2017).

La lutte chimique est une des solutions au problème phytosanitaire du niébé, mais aussi la disponibilité des pesticides de bonne qualité est une contrainte pour ces zones qui sont en retrait par rapport aux grandes villes. De plus, le faible revenu des producteurs ne leur permet pas d'acquérir ces pesticides souvent importés d'Europe.
C'est pourquoi l'utilisation du biopesticide semble être une alternative intéressante. Dans la présente étude, le jus de graines de Neem a permis une réduction importante du nombre des insectes ravageurs et aussi des dégâts qui leur sont associés. Une étude similaire sur le niébé a démontré que les feuilles de Neem à la dose de $1 \mathrm{~kg}$ pour 6 litres d'eau pulvérisées sur $200 \mathrm{~m}^{2}$ a réduit les dégâts des punaises de 37,9\% au Burkina Faso (Bambara et Tiemtoré, 2008).

Le rendement obtenu de l'ordre de 980 $\mathrm{kg} / \mathrm{ha}$ est au moins le double de la moyenne nationale. Ces données démontrent les vertus du Neem comme pesticide tel que rapporté par plusieurs auteurs (Biswas, 2013 ; Campos et al., 2016). L'efficacité des extraits de graines de Neem contre les thrips et $M$. vitrata a déjà été rapportée par de nombreux auteurs (Irulandi et Balasubramanian, 2000 ; Tanzubil, 2000 ; Oparaeke, 2006). Gauvin et al. (2002) ont montré que l'azadirachtine, principale composante à propriétés insecticides des extraits de Neem est concentré dans les graines et les huiles. Celle-ci serait responsable de l'action sur les insectes des fleurs du niébé grâce à ses propriétés anti-appétantes, répulsives et régulateurs de croissance sur diverses espèces d'insectes (Belanger et Musabyimana, 2005 ; Isman, 2006).

Cependant, il a été noté que l'huile de Neem a été moins performante que le jus de graines de Neem. Cette situation pourrait être expliquée par le taux d'azadirachtine et ses dérivés plus élevés dans le jus de graine de Neem préparé à partir de graines directement écrasées (Isman, 2006).

Le virus MaviNPV seul ou associé au Neem a permis une réduction des populations et des dégâts de $M$. vitrata. L'efficacité de cette combinaison a été testée au Bénin (Mehinto et al., 2014). Il a été obtenu un rendement de niébé de plus de $800,0 \mathrm{~kg} / \mathrm{ha}$. Les résultats peu encourageants obtenus au cours de l'année 2015 au Niger pourront être expliqués par la faible densité des larves de $M$. vitrata et la forte densité de $C$. tomentosicollis, insecte non affecté par le virus MaviNPV. 


\section{Conclusion}

La présente étude nous a permis de comparer l'efficacité de plusieurs biopesticides. Il est ressorti que l'utilisation des extraits aqueux des graines de Neem à la dose de 5\% (500 g de graines de Neem pour 10 litres d'eau) a réduit les infestations et les dégâts du ravageur tout en permettant d'obtenir un rendement acceptable de niébé de 980,0 kg/ha en deux années d'expérimentation. L'utilisation de cette technologie est à promouvoir en milieu paysan comme solution de lutte alternative contre les principaux insectes ravageurs du niébé. Les producteurs doivent être sensibilisés pour leur permettre d'utiliser le biopesticide sur les jeunes stades des ravageurs avant la phase de dégâts.

\section{CONFLITS D'INTERETS}

Les auteurs déclarent qu'aucun intérêt n'est en compétition dans cet article.

\section{CONTRIBUTIONS DES AUTEURS}

MAH a conçu le protocole, collecté les données et rédigé l'article. IB a supervisé l'étude et la rédaction scientifique. SL, MT, LA, SM et BP ont contribué à la correction et à la finalisation du manuscrit.

\section{REMERCIEMENTS}

Les auteurs remercient le projet "Promotion des biopesticides pour la gestion intégrée des insectes ravageurs du niébé en champ au Niger et au Burkina Faso" qui a financé cette étude dans le cadre du Programme de Productivité Agricole en Afrique de l'Ouest (PPAAO). Les auteurs remercient également le projet Legume-Lab Innovation financé par l'US Agency for International Development (USAID) pour avoir soutenu matériellement les étudiants impliqués dans la collecte des données de terrain et tous les chefs des villages visités au cours des déplacements pour leur accueil chaleureux. Enfin, les auteurs remercient vivement les examinateurs pour leurs contributions à l'amélioration du manuscrit.

\section{REFERENCES}

Abdoulaye OZ, Baoua I, Boureima S, Amadou L, Tamo M, Mahamane S, Mahamane A, Pittendrigh BR. 2018. Etude de l'efficacité des biopesticides dérivés du Neem et de l'entomopathogene MaviNPV pour la gestion des insectes ravageurs du niébé au Niger. Bulletin de la Recherche Agronomique du Bénin (BRAB), (83): 16-24.

Abdourahamane HM, Baoua I, Tamo M, Amadou L, Mahamane S, Pittendrigh B. 2018. Etude des paramètres de reproduction et de développement de Clavigralla tomentosicollis Stål, 1855 (Hemiptera : Coreidae) et son incidence sur le rendement du niébé dans la région de Maradi au Niger. REV. RAMRES, Science de la Vie, de la Terre et Agronomie, 6(1): 42- 48.

Ahmed BI, Onu I, Mudi L. 2009. Field bioefficacy of plant extracts for the control of post flowering insect pests of cowpea (Vigna unguiculata (L.) Walp.) in Nigeria. Journal of Biopesticides, 2(1): 37- 43.

Ameh SA, Ogunwolu EO. 2000. Comparative effectiveness of aqueous plant extracts and lambda cyhalothrin in controlling post flowering insect pests of cowpea in the Southern Guinea savanna of Nigeria. In Entomology in nation building: the Nigerian experience. The Proceedings of ESN 30th Annual Conference held at Kano, Nigeria, 4th-7th October 1999 Entomological Society of Nigeria; 175180.

Asiwe JAN. 2006. Baseline survey on the production practices, constraints and utilization of cowpea in South Africa: implications for cowpea improvement. In International Conference on Indigenous Vegetables and Legumes. Prospectus for Fighting Poverty, Hunger and Malnutrition 752; 381-385.

Bambara D, Tiemtoré J. 2008. Efficacité biopesticide de Hyptis spicigera Lam., Azadirachta indica A. Juss. et Euphorbia balsamifera Ait. sur le niébé Vigna unguculata L. Walp. Tropicultura, 26(1): 53-55. 
Bass C, Denholm I, Williamson MS, Nauen R. 2015. The global status of insect resistance to neonicotinoid insecticides. Pesticide Biochemistry and Physiology, 121 : 78-87.

Bélanger A, Musabyimana T. 2005. Le Neem contre les insectes et les maladies. Journées Horticoles au Canada en 2005.

Biswas GC. 2013. Comparative effectiveness of Neem extracts and synthetic organic insecticide against mustard aphid. Bangladesh Journal of Agricultural Research, 38(2): 181-187.

Campos EV, de Oliveira JL, Pascoli M, de Lima R, Fraceto LF. 2016. Neem oil and crop protection: from now to the future. Frontiers in Plant Science, 7: 1494.

Carlos JSP. 2006. «Exposition humaine aux pesticides: un facteur de risque pour le suicide au Brésil ». Vertigo-La revue en Science de l'Environnement ; 7-18.

Cisse N, Hall AE. 2002. La culture traditionnelle du niébé au Sénégal. ISRA, CNRA. Bambey Sénégal. p. 37.

Deguine JP, Ferron P. 2004. Protection des cultures et développement durable : bilan et perspectives. Courrier de l'Environnement de l'INRA, (52) : 57- 65.

Dugjé IY, Omoigui LO, Ekeleme F, Kamara AY, Ajeigbe H. 2009. Production du niébé en Afrique de l'Ouest : Guide du paysan. IITA, Ibadan, Nigeria, p. 20.

Egho EO, Ilondu EM. 2012. Seeds of Neem Tree (Azadirachta indica A. Juss). Promising Biopesticide in the Management of Cowpea Insect Pests and Grain Yield in the Early Cropping Season at Asaba and Abraka, Delta State, Nigeria. Journal of Agricultural Science, 4(1): 181-189.

Egho EO. 2011. Evaluation of Neem Seed Extract for the Control of Major Field Pests of Cowpea (Vigna unguiculata L. Walp) under Calendar and Monitored Sprays. Advances in Environmental Biology, 5(1): 61-66. DOI: http://dx.doi.org/10.5539/jas.v4n1p181

Facknath S. 2006. Combination of Neem and physical disturbance for the control of four insect pests of stored products. International Journal of Tropical Insect
Science, 26(1): 16-27. DOI: https://doi.org/10.1079/IJT200698

FAO. 2004. COWPEA: Post - Harvest Operations, 71 p. Retrouvé sur le web le $16 / 07 / 2018$ à l'adresse : http://www.fao.org/3/a-au994e.pdf

Gauvin MJ, Bélanger A, Nébié R, Boivin G. 2003. Azadirachta indica: l'azadirachtine est-elle le seul ingrédient actif?. Phytoprotection, 84(2): 115-119. DOI: 10.7202/007814ar

Gomgnimbou APK, Savadogo PW, Nianogo AJ, Millogo-Rasolodimby J. 2009. Usage des intrants chimiques dans un agrosystème tropical : diagnostic du risque de pollution environnementale dans la région cotonnière de l'est du Burkina Faso. Biotechnologie, Agronomie, Société et Environnement, 13(4) : 499-507.

Habiba Y. 2004. Evaluation agronomique de quelques variétés de niébé pour la production de graines et des fanes et leur résistance aux principaux ennemis. Mémoire de Maîtrise, Université de Niamey, Niger, $\quad$ p. 32. https://doi.org/10.1016/j.pestbp.2015.04. 004

Irulandi S, Balasubramanian G. 2000. Report on the effect of botanicals against Megalurothrips distalis K. (Tripidae: Thysanoptera) and Lampides boeticus Linn. (Lycaenidae: Lepidoptera) on greengram. Insect Environment, 5(4): 175-176.

Isman MB. 2006. Botanical insecticides, deterrents, and repellents in modern agriculture and an increasingly regulated world. Annual Review of Entomology, 51: 45-66.

DOI: 10.1146/annurev.ento.51.110104.151146

Liao CT, Lin CS. 2000: Occurrence of legume podborer Maruca testulalis (Geyer) (Lepidoptera: Pyralidae) on cowpea Vigna unguiculata (L.) Walp. and its insecticides application trial. Plant Protection Bulletin-Tapei, 42(4): 213222.

MA. 2018. Rapport d'évaluation de la campagne agricole d'hivernage 2017 et perspectives alimentaires 2017-2018. 
Direction générale des ressources, direction des statistiques, p. 42.

Mehinto JT, Atachi P, Elégbédé M, Kpindou OKD, Tamò M. 2014. Efficacité comparée des insecticides de natures différentes dans la gestion des insectes ravageurs du niébé au Centre du Bénin. Journal of Applied Biosciences, 84(1): 7695-7706.

DOI: http://dx.doi.org/10.4314/jab.v84i1.1

Mominé B. 2014. Etude de l'efficacité de l'extrait aqueux de graines de Neem et la deltaméthrine sur les insectes ravageurs du pourghère (Jatropha curcas L.): cas de Calidea dregii Germar. et Aphtona spp. Mémoire de fin de cycle en vue de l'obtention du diplôme de Master en production végétale, Université Polytechnique de Bobo-Dioulasso (U.P.B.), p. 52.

Mouffok B, Raffy E, Urruty N, Zjcola J. 2008. Le Neem, un insecticide biologique efficace. Université Paul Sabatier IUT, S2, p. 16.

N'gbesso MFP, Zohouri GP, Fondio L, Djidji AH, Konate D. 2013. Etude des caractéristiques de croissance et de l'état sanitaire de six variétés améliorées de niébé [Vigna unguiculata (L)] en zone centrale de Côte d'Ivoire. International Journal of Biological and Chemical Sciences, 7(2): 457-467. DOI: http://dx.doi.org/10.4314/ijbcs.v7i2.4

Narayan S, Liew Z, Bronstein JM, Ritz B. 2017. Occupational pesticide use and Parkinson's disease in the Parkinson Environment Gene (PEG) study. Environment international, 107: 266-273. https://doi.org/10.1016/j.envint.2017.04. 010

Oparaeke AM. 2006. Effect of aqueous extracts of tropical plants for management of Maruca vitrata Fab. and Clavigralla tomentosicollis Stal. on cowpea, Vigna unguiculata (L.) Walp. plants. Journal of Entomology, 3 (1): 7075.

Ouédraogo E. 2004. L'utilisation des insecticides naturels dans la protection des cultures au Burkina Faso. Communication faite au CTR de
l'INERA Di. 20-22 déc. 2004 Ouagadougou CEAS, p. 56.

Oyewale RO, Bamaiyi LJ. 2013. Management of cowpea insect pests. Scholars Academic Journal of Biosciences, 1(5): 217-226.

Rabé MM, Ibrahim B, Razack A, Lawali S, Laouali A, Barry P, Saadou M. 2017. Les déterminants socioéconomiques de l'adoption des technologies améliorées de production du niébé diffusées par les champs écoles paysans dans les régions de Maradi et Zinder au Niger. International Journal of Biological and Chemical Sciences, 11(2): 744 -756. DOI: https://dx.doi.org/10.4314/ijbcs.v11i2.17

Reddy NP, Khan APA, Uma DK, Victor SJ, Sharma HC. 2008. Assessment of the suitability of Tinopal as an enhancing adjuvant in formulations of the insect pathogenic fungi Beauveria bassiana (Bals.) Vuillemin. Pest Management Science: formerly Pesticide Science, 64(9): 909-915. DOI: https://doi.org/10.1002/ps.1581

Sabbour M, Sahab AF. 2005. Efficacy of some microbial control agents against cabbage pests in Egypt. Pakistan Journal of Biological Sciences, 8 (10): 1351-1356. DOI: $10.3923 /$ pjbs.2005.1351.1356

Sankoh AI, Whittle R, Semple KT, Jones KC, Sweetman AJ. 2016. An assessment of the impacts of pesticide use on the environment and health of rice farmers in Sierra Leone. Environment International, 94: 458-466. DOI : https://doi.org/10.1016/j.envint.2016.05. 034

Sokamé MB. 2012. Etude des interactions entre le baculovirus MaviNPV, les huiles de Neem (Azadirachta indica Juss) et de pourghère (Jatropha curcas L.) pour la lutte contre trois principaux ravageurs du niébé, Vigna unguiculata (L). Walp. (Fabales : Fabaceae). Mémoire d'ingénieur : ESA/UL, 106. p.

Srinivasan R, Tamo M, Lee ST, Lin MY, Huang CC, Hsu YC, Su FC. 2008. Developing an integrated pest management (IPM) strategy for the legume pod borer (Maruca vitrata). In: 
Proceedings of the International Congress of Entomology 2008, Durban, South Africa. p. 621. http://www.ice2008.org.za/pdf/proceedin gs.pdf

Talekar N. 2006. Eléments de cours en défense des cultures: Pest management in soybean and cowpea cultivation. Treizième cours régional sur la recherche et la production des plantes légumières, 01 Juillet au 06 Octobre 2006 en Tanzanie, AVRDC, Arusha-Tanzania, p. 54.

Tamò M, Ekesi S, Maniania NK, Cherry A. 2003. Biological control, a non-obvious component of integrated pest management for cowpea. Biological control in integrated pest management systems in Africa, 295-309.

Tamo M, Goergen G, Agboton C, Srinivasan R. 2007. Putting agro-biodiversity to work: the cowpea story. In: Proceedings of XVI International Plant Protection Congress. 354-355.

Tanzubil PB, Zakariah M, Alem A. 2008. Integrating host plant resistance and chemical control in the management of Cowpea pests. Australian Journal of Crop Science, 2(3): 115-120.
Tanzubil PB. 2000. Field evaluation of Neem, Azadirachta indica extracts for control of insect pests of cowpea in Northern Ghana. Journal of Tropical Forest Products, 6(2): 165-172.

Tonessia C, Wade M, Cissé N, Aké S. 2009. Caractérisation de Striga gesnerioides (Willd.) Vatke du Sénégal : Réactions de plusieurs cultivars de niébé (Vigna unguiculata L. Walp.). Journal of Applied BioSciences, 24(7): 1462-1476.

Vinod K, Singh NP. 2009. Spodoptera litura Nuclear Polyhedrosis Virus (NPV-S) as a component in integrated pest management (IPM) of Spodoptera litura (Fab.) on cabbage. Journal of Biopesticides, 2(1): 84-86.

Zakari AH, Mahamadou CI, Hachimou Z, Toudou A. 2016. Efficacité de l'huile de Neem (Azadirachta indica) et de Bacillus thuringiensis (Biobit 2X) sur la dynamique de la population de Bemisia tabaci (Gennadius 1889) et Helicoverpa armigera (Hubner, 1808) dans une plantation de tomate au Niger. International Journal of Biological and Chemical Sciences, 10(2): 497-505. DOI: http://dx.doi.org/10.4314/ijbcs.v10i2.4. 